\title{
Estimation of the spanwise wall shear stress based on upstream information for wall turbulence control
}

\author{
B. Frohnapfel*, Y. Hasegawa ${ }^{+}$, N. Kasagi ${ }^{+}$, S. Große $^{o}$ \\ * Center of Smart Interfaces, TU Darmstadt, Germany \\ + Dept. of Mechanical Engineering, The University of Tokyo, Japan \\ ${ }^{\circ}$ Lab. Aero- and Hydrodynamics, Delft Univ. of Technology, The Netherlands \\ frohnapfel at csi.tu-darmstadt.de
}

In the last decade many research efforts in flow control were directed to feedback control schemes due to the fact that large energy gain was anticipated. To achieve practically realizable control, the footprints of near wall turbulence need to be detected through sensors that are mounted on the wall. In this respect, sensing wall shear stresses is considered most feasible. Especially the instantaneous spanwise wall shear stress, $\tau_{z}=(\partial w / \partial y)_{\text {wall }}$, is known to be useful for the state estimation of wall turbulence and it has been shown that control laws based only on the time trace of this component work well $[1,2]$.

Up to now, most of the research on feedback control have been done in direct numerical simulation (DNS) with idealized systems in which continuous sensing and actuation are assumed at the same location. Since this assumption is unrealistic, we investigate whether the local instantaneous spanwise wall shear stress can be obtained based on upstream information.

It is well known that quasi streamwise vortices (QSV) as well as high and low speed streaks are a part of the regeneration cycle of wall turbulence [3]. Endo et al. [4] showed that the QSV located at the downstream side of a meandering low speed streak can be captured by measuring the spatial gradients of both wall shear stresses, $\tau_{x}=(\partial u / \partial y)_{\text {wall }}$ and $\tau_{z}=(\partial w / \partial y)_{\text {wall }}$, about 50 viscous length units upstream. In general, the experimental assessment of $\tau_{z}$ and its spatial gradients needs significantly more effort than the measurement of $\tau_{x}$ [5]. With the goal of providing an easily accessible input information that replaces the knowledge of $\tau_{z}$ at the actuation location, we investigate the correlation between the spanwise gradient of the streamwise wall shear stress, $\partial \tau_{x} / \partial z=\frac{\partial}{\partial z}(\partial u / \partial y)_{w a l l}$, and $\tau_{z}$.

In general, the correlation function has a form of $R(\Delta x, \Delta z, \Delta t)$. The present work is limited to the case where the time lag, $\Delta t$, and the spanwise displacement, $\Delta z$, are zero. 

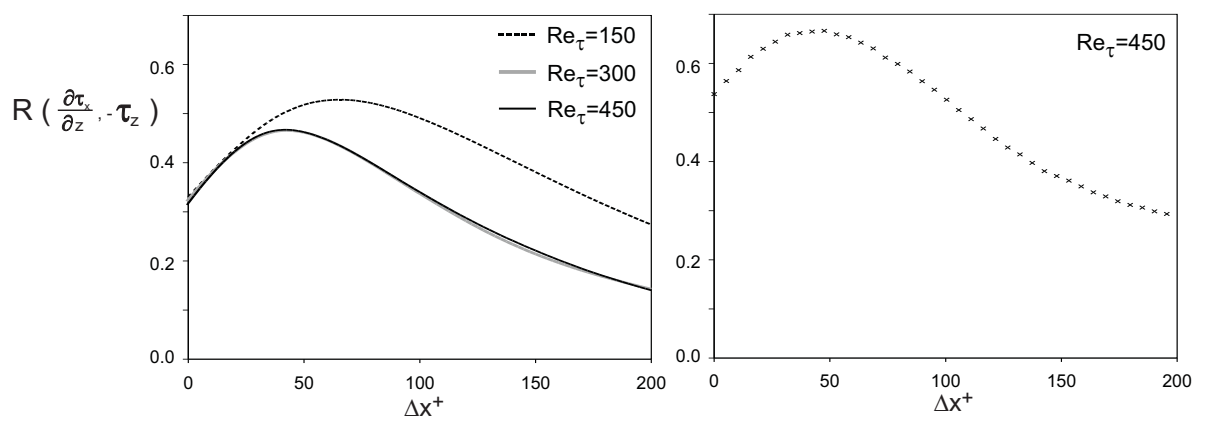

Fig. 1. Spatial correlation between the spanwise gradient of the streamwise wall shear stress and the negative wall shear stress in a turbulent channel flow (left, DNS) and a duct flow (right, experiment).

We carry out DNS of a fully developed channel flow with a constant flow rate. The bulk Reynolds number based on the channel height is changed as $R e_{b}=U_{b} H / \nu \simeq 4600,10100$ and 16100, which corresponds to friction Reynolds numbers for the uncontrolled case, $R e_{\tau}=u_{\tau} \delta / \nu=150,300$ and 450, respectively.

The obtained spatial correlations, $R\left(\frac{\partial \tau_{x}}{\partial z}(x),-\tau_{z}\left(x+\Delta x^{+}\right)\right)$, for the three Reynolds numbers are depicted in Fig. 1, left. For $R e_{\tau}=150$ the highest correlation is slightly above $50 \%$ and is located at a streamwise distance of $\Delta x^{+} \approx 65$. For $R e_{\tau}=300$ and 450 the maximum value is reduced to $47 \%$ and shifted to smaller streamwise distances such that the maximum correlation is located at $\Delta x^{+} \approx 43$. The good agreement between the two correlations at the higher Reynolds numbers suggests that the Reynolds number dependence at $R e_{\tau}>300$ would be small.

In addition to the numerical simulation, we consider experimental data obtained in a duct flow at $R e_{b} \simeq 15400$. The corresponding friction Reynolds number is given by $R e_{\tau}=u_{\tau} \delta / \nu=450$. A spanwise row of micro-pillar shear-stress sensors MPS $^{3}$ [6], shown in Fig. 2, is placed in the duct flow to simultaneously measure the instantaneous streamwise and spanwise wall shear stresses. The sensors do not emerge out of the viscous sublayer and measure the temporal evolution of the wall shear stresses such that a temporal correlation between $\partial \tau_{x} / \partial z$ and $\tau_{z}$ can be obtained in the center of the duct where the flow was shown to be two-dimensional based on PIV data. In the present investigation the spanwise spacing of the sensors is given by $\Delta z^{+}=10$. Employing Taylor's Hypothesis the temporal correlation is transformed to the spatial correlation.

The resulting correlation is plotted in Fig. 1, right. It can be seen that the correlation for the duct flow shows the same qualitative trend as the correlations obtained for channel flows at $R e_{\tau}=300$ and 450, suggesting that the convection velocity was chosen appropriately. However, the correlation values are much higher than in the channel flow simulation. The difference between 


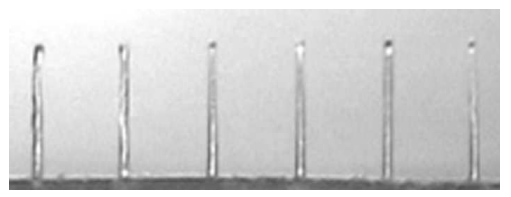

Fig. 2. A row of micro-pillar shear-stress sensors. If the sensors are aligned in spanwise direction $(\mathrm{z})$ it is possible to obtain a temporal correlation between $\partial \tau_{x} / \partial z$ and $\tau_{z}$. Note that in the present investigation the sensors are placed further apart than in the picture. Their spacing roughly corresponds to twice the pillar height.

the obtained correlations cannot solely be attributed to the statistical uncertainty in the available data. Since the evaluation of $\partial \tau_{x} / \partial z$ in the experimental investigation is based on the spanwise distance between the pillars, we have performed numerical reference computations in which the spanwise distance of the $\tau_{x}$-sensing locations is increased up to two pillar spacings. We found that this increased "sensor spacing" does not significantly influence the correlation value. At this moment we can not explain the observed difference, although it seems that the temporal correlation is higher than the spatial one.

In order to test the feasibility of the suggested $\tau_{z}$-estimation, we investigate the influence of replacing $\tau_{z}$ with the upstream information of $\partial \tau_{x} / \partial z$ in a control loop similar to the one suggested in [2]. We employ a spanwise body force within a forcing layer $y_{f}^{+}=10$ on the top and bottom walls of the channel flow and carry out simulations at $R e_{\tau}=150$ in which the body force is given by either

$$
b f(x, y, z)=\frac{1}{\Phi}\left(y_{f}-y\right) \underbrace{\tau_{z}(x, z)}_{\text {measured quantity }}
$$

or

$$
b f(x, y, z)=\frac{1}{\Phi}\left(y_{f}-y\right) \underbrace{\frac{\partial \tau_{x}(x-\Delta x, z)}{\partial z}}_{\text {measured quantity }} .
$$

Note that $\Delta x$ corresponds to the streamwise distance between sensor and actuator locations and that $\Phi$ is the forcing time constant which, in combination with the sensor value, determines the strength of the forcing and thus the required control power input.

Based on the body force formulation given by equation (1) drag reduction of $D R=25.3 \%$ can be achieved. With the body force formulation given by equation (2) and $\Delta x^{+}=65$ (location of the highest correlation) a similar control power input leads to $D R=17.2 \%$.

Fig. 3 shows the obtained Reynolds stresses in comparison to the uncontrolled flow. It can be seen that the two control schemes have a very similar influence on $\overline{u_{2}^{2}}, \overline{u_{3}^{2}}$ and $\overline{u_{1} u_{2}}$. The suppression of these components is stronger for higher drag reduction as expected. For $\overline{u_{1}^{2}}$ this is not the case, and this 

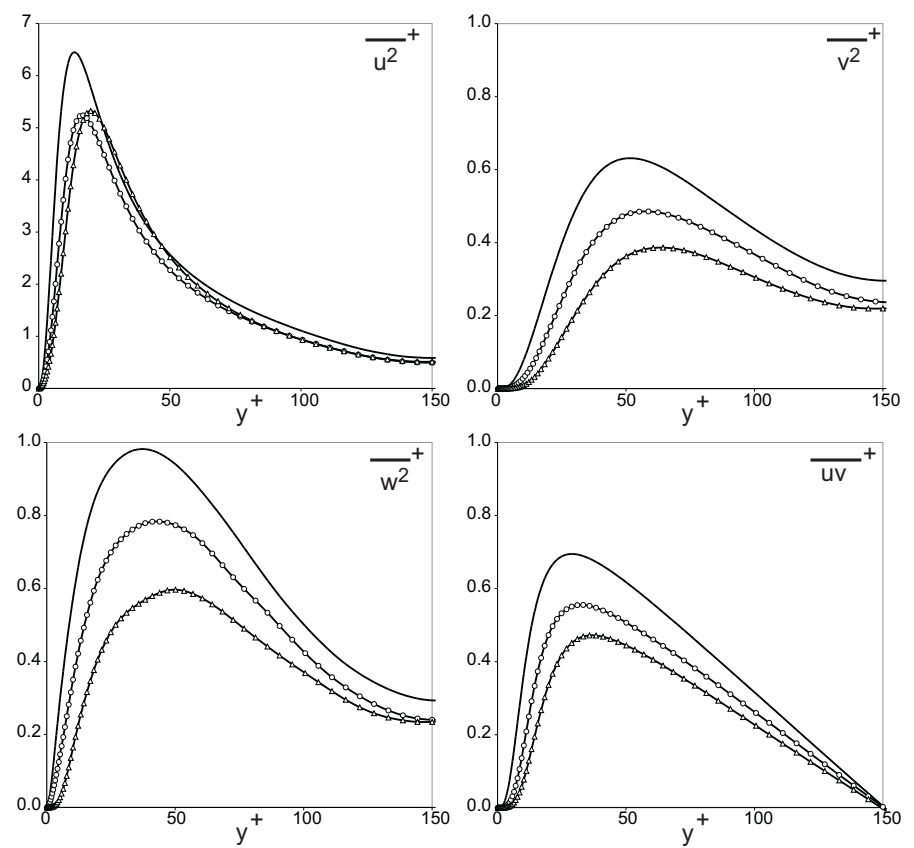

Fig. 3. Reynolds stress distribution for the control based on equation (1) (triangles, $\mathrm{DR}=25.3 \%$ ) and on equation (2) (circles, $\mathrm{DR}=17.2 \%$ ) in comparison to the uncontrolled channel flow (straight line). The normalization is based on the inner variables of the uncontrolled channel flow.

fact suggests that the suppression of $\overline{u_{1}^{2}}$ is an effect that consumes energy but is not necessarily required for drag reduction. If the sensor is placed at $\Delta x^{+}=47, D R=16.3 \%$ is achieved.

The present results demonstrate that the upstream spanwise gradient of the streamwise wall shear stress, $\partial \tau_{x} / \partial z$, can be used to predict the spanwise wall shear stress which is known to be a good candidate for state estimation of near-wall turbulence. It was shown that this correlation does not only exist in uncontrolled flows but that it can also be successfully used for the design of flow control loops, at least at low Reynolds numbers. Whether this also holds at higher Reynolds numbers remains to be clarified in future work.

\section{References}

1. C. Lee, J. Kim, H. Choi, J. Fluid Mechanics, 358, 245 (1998).

2. C. Lee, J. Kim, Physics of Fluids, 14(7), 2523 (2002).

3. J.M. Hamilton, J. Kim, F. Waleffe, J. Fluid Mechanics, 287, 317 (1995).

4. T. Endo, N. Kasagi, Y. Suzuki, Int. J. Heat Fluid Flow, 21, 568 (2000).

5. Y. Suzuki, N. Kasagi, Experimental Thermal and Fluid Science, 5, 69 (1992).

6. S. Große, W. Schröder, AIAA Journal, 47(2), 314 (2009). 